\title{
H-ALPHA AND OI 7774 SPECTROSCOPY OF ALGOL-TYPE BINARIES
}

\author{
Paul B. Etzel \\ Department of Astronomy \\ San Diego State University \\ San Diego, CA 92182 USA \\ Edward C. Olson \\ Department of Astronomy \\ University of Illinois \\ Urbana, IL 61801 USA
}

\begin{abstract}
Spectroscopic observations are presented showing evidence of mass transfer activity in Algol-type eclipsing binaries. Traditional $\mathrm{H} \alpha$ emission line spectroscopy is discussed along with absorption line spectroscopy of the O I $7774 \mathrm{~A}$ multiplet. Eclipse effects in both lines are discussed, along with a new non-LTE model for Hydrogen line spectrum synthesis.
\end{abstract}

\section{Introduction}

For most Algol-type binaries, the hotter (primary) component is usually the smaller and more massive of the pair; it is much like a normal main-sequence star. The cooler (secondary) component is usually overluminous for its mass. By definition, the secondary component of a true Algol fills its critical Roche lobe; mass transfer to the primary component is therefore possible. The velocity curves of the primaries of Algols frequently show complications as a result of circumstellar matter (Popper 1989).

Joy (1942) established the basic methodology, and model framework, for the study of mass transfer, in various types of interacting binaries. The mass-transfer process can lead to the formation a luminous ring or "accretion disk" in rotation about the primary component. The profiles of the Balmer lines (frequently seen in emission) have historically provided a readily observable diagnostic of circumstellar matter (Kaitchuck, Honeycutt \& Schlegel 1985). The approaching and receding portions of an accretion disk are successively occulted about primary stellar eclipse to produce the so-called "V/R" variation seen in the Balmer emissions (e.g., Peters 1989). In longer period systems, the disks can become large enough (hence luminous enough) to be observed outside of eclipse, as shown in Fig. 1 for KU Cygni ( $\mathrm{P}=38.4$ days) at $\mathrm{H} \alpha$.

\section{Observational Program}

For the past three years, the authors have been observing Algol-type and related systems, with orbital periods ranging from 4 to 262 days. Nearly simultaneous spectroscopic and 
photometric observations are made with the $1.0 \mathrm{~m}$ reflector at Mount Laguna Observatory, operated jointly by San Diego State University (SDSU) and the University of Illinois, Urbana-Champaign (UI). In ten minutes, we can switch between a CCD spectrograph, which provides a resolution of about $1.2 \mathrm{~A}$, and an automated photometer. Both instruments are mounted on the telescope; the operational one is selected by flip mirrors.

Olson's long-term photometric program of 5-color photometry (uvbyI), has been augmented with two additional filters at $6870 \mathrm{~A}$ (r) and $7950 \mathrm{~A}$ (i) designed by Etzel. The photometry is employed to: 1) obtain fundamental stellar properties, such as radii, to provide key geometrical scale information for the accretion disk, 2) look for evidence of mass-transfer induced distortions in the light curve, and 3) determine the relative stellar luminosities over a wide range of wavelengths. Spectra are obtained at well sampled phase intervals of the stellar orbits to study the distribution of circumstellar matter. In addition to observations at $\mathrm{H} \alpha$ (occasionally $\mathrm{H} \beta$ ), many spectra have been obtained of the O I $7774 \mathrm{~A}$ multiplet, which is seen in absorption in normal Algols.

Etzel $(1986,1988)$ demonstrated for the system TT Hydrae $(\mathrm{P}=6.95$ days $)$ that the majority of the metallic absorption lines seen in the optical region of the spectrum arise from an extended shell (circumstellar matter in a dilute radiation field) about the more luminous primary and not its photosphere. These metallic shell lines (including O I 7774 multiplet) are actually too strong because they arise from low-lying metastable states that are collisionally populated. Previous spectral classifications for the primary component of TT Hya ranged from A2V to A5III! Such misclassifications were largely due to the perceived weakness of Si II and Mg II lines, which are actually photospheric lines of normal strength. The mass, radius, and spectral-energy distribution (SED) of the primary star are all consistent with a B9.5 V star. However, a $40 \mathrm{~A}$ wide flux point centered at $7780 \mathrm{~A}$ in the SED of the hotter star is well below the level anticipated for a normal photosphere (blocking factor of $4.8 \mathrm{~A}$ ). High-dispersion spectroscopy has revealed the OI 7774 multiplet to be abnormally strong, which is consistent with the metallic shellspectrum interpretation (Polidan and Peters 1976, Slettebak 1986).

The non-photospheric nature of the O I multiplet in TT Hya is illustrated in Fig. 2. At this wavelength, the hotter component accounts for $55 \%$ of the light of the system. This component is very similar to the B9.5 V spectral standard, $\theta \mathrm{Crt}$. If the $\mathrm{O}$ I absorption in TT Hya were simply dominated by the hotter component, its measured (continuum diluted) equivalent width should only be about $0.36 \mathrm{~A}$. The increased absorption is due to circumstellar material (accretion disk), which must cover a geometrically large fraction of the apparent stellar disk (see also Etzel's 1986, 1988 arguments for the Ca II $\mathrm{K}$ and $\mathrm{Na} I \mathrm{D}$ lines). The absorption increases at phases nearer secondary eclipse as the accretion disk is projected across the much larger secondary star. There is also a corresponding increase in $\mathrm{H} \alpha$ central absorption at secondary eclipse phases and also a noticeable contribution from the stellar absorption produced by the secondary component (Etzel 1986, 1988, 1990).

The $\mathrm{O}$ I multiplet has proven to be a useful diagnostic for the study of the distribution of circumstellar matter in Algol and related systems. Unlike $\mathrm{H} \alpha$, the profile of which is an integrated measure of the accretion-disk material at various locations and velocities, absorption-line spectroscopy provides a measure along a relatively simple column in the line of sight to the photosphere of the hotter star that can, in theory, be used to construct a two dimensional picture of the distribution of matter within the disk. At the start of our observing project, the $\mathrm{O}$ I multiplet was selected for study because of the relatively low resolution of our spectrograph. However, future studies of metallic-shell absorptions, with a higher dispersion spectrograph, could prove more fruitful to test various velocitydispersion hypotheses (Etzel 1986, 1988). 
Fig. 3 dramatically illustrates the usefulness of the $O$ I multiplet for delineating the extent of the accretion disk within the long-period system RZ Ophiuchii ( $\mathrm{P}=262$ days). At phases outside eclipse, the absorption produced by circumstellar material surrounding the smaller, mass-gaining star is minimal. Absorption rapidly increases in strength as the disk increasingly occults the much larger, cooler, and, at this wavelength, almost equally luminous component. Equivalent widths for O I 7774 as high as $5 \mathrm{~A}$ have been observed in KU Cyg near secondary eclipse!

SDSU graduate student Senay (1991) has completed a spectroscopic survey of the O I 7774 feature in 135 binary systems of known or suspected Algol-type (Budding 1984). $\mathrm{He}$ found abnormal $\mathrm{O}$ I strength to be a good indicator of the presence of circumstellar matter in binary systems. He was able to demonstrate the existence of circumstellar matter in about twice as many cases than with $\mathrm{H} \alpha$ alone. Typically, such detections were in situations where $\mathrm{H} \alpha$ emission might be only marginally detectable at eclipse totality.

\section{The Approximate non-LTE Model for Hydrogen Emission Lines}

The circumstellar accretion disks discussed above differ from most disks around compact objects. Continua (except the Lyman) are optically thin, so standard alpha-disk theory does not apply. Surface elements on the disk do not radiate as independent stellar atmospheres, so the usual non-LTE techniques also do not apply. Olson (1991) has developed an approximate non-LTE disk model using several approximations to enable us to calculate $H \alpha$ and $H \beta$ emission profiles from such disks. These models are spectroscopic diagnostic tools, and not self-consistent physical models.

Emission-line formation in LTE would be appropriate for $\mathrm{H} \alpha$ if bound-bound and bound-free transitions among the lowest levels were nearly opaque, and therefore close to detailed radiative balance (DRB). Moreover, high opacity would have to be reached over geometrically small distances compared to the disk radius (to preserve the 'local' requirement), and be achieved in the presence of Doppler shifts from differential disk rotation. Calculations at various points in model disks show that differential rotation does not render optically thin any point where a Balmer-line optical depth is large in the vertical direction. This last circumstance is a key simplification in this model.

Major non-LTE effects have been treated by recognizing 'excitation regimes' in which various hydrogen transitions are nearly opaque, and therefore close to DRB. These radiative rates then subtract out of the equations for statistical equilibrium. Over relevant parts of the disk, all Lyman transitions satisfy this condition. At a fixed disk radius and with decreasing height, $\mathrm{H} \alpha, \mathrm{H} \beta$, and $\mathrm{H} \gamma$ may become opaque in succession. The Balmer continuum remains optically thin. Balmer line and continuum opacities are set by $n 2(\mathrm{H})$, which is nearly independent of the precise excitation conditions that determine $\mathrm{n} 3(\mathrm{H}) / \mathrm{n} 2(\mathrm{H})$, and therefore the line source function. Excitation regimes also recognize that a point in the disk may be illuminated by optically thick Balmer-line radiation from the central plane of the disk. Separate statistical equilibrium calculations, using a 5-level model hydrogen atom, are made for each excitation condition; $n 3(\mathrm{H}) / \mathrm{n} 2(\mathrm{H})$ and the nonLTE source function then follow. The model includes:

1. hydrostatic equilibrium in the $\mathrm{z}$-direction (height).

2. kinetic temperature a linear (or other) function of disk radial position.

3. temperature constant or a linear function of height.

4. pressure an exponential (or other) function of radial position.

5. Keplerian or non-Keplerian disk rotation.

6. microturbulence. 
The integration grid consists of columns $d x^{*} d z$ in the line-of sight $(y)$ covering half the disk. Finer dz (height) steps are used close to the orbital plane, where gravitational stratification leads to the highest column intensities. Up to 100 columns are needed for accurate profiles. In a given column through the disk, the optical depth remains small until the total radial velocity of hot star and disk rotation shifts the hydrogen-line coefficient close to the wavelength being treated. Thereafter, the line optical depth can quickly rise to several hundred (in which case the emergent intensity is taken as the source function at unit optical depth). An adaptable grid is required along the line-ofsight, to handle such rapid opacity changes. Contributions to the emitted profile derive from most of the disk interior, because of changes in disk radial velocity over the $\mathrm{dx}-\mathrm{dz}$ grid. Thus, information from much of the disk volume should be contained in the $\mathrm{H} \alpha$ emission profile, making it possible, in principle, to probe the physical structure over most of that volume. Partial eclipses of the disk by the large secondary star also should sharpen this probe. This favorable property of large low-density disks in non-degenerate binaries probably does not apply to denser disks in degenerate binaries, where continuous opacity is usually large and emission line formation therefore is mostly a 'skin effect'.

Sample calculations have been done for the relatively cool disks that may surround late A or F mass-gaining stars. Non-LTE H $\alpha$ luminosities exceed LTE values by a factor of about 3 , and are strong enough to be easily visible at all orbital phases, which is in qualitative agreement with observations. In LTE, $H \alpha$ and $H \beta$ luminosities are comparable, while in non-LTE, H $\alpha$ is much stronger -- again in qualitative agreement with observations. Disk continuum emission is weak compared to line emission, which agrees with photometric observations of these binaries. Non-LTE H $\alpha$ profiles are very sensitive to kinetic temperature and to departures from Keplerian rotation. Profiles during the progressive eclipse of the disk by the cool star should probe the radial dependencies of physical disk parameters. Calculations show that the $\mathrm{H} \alpha$ light lost (not left) in such an eclipse is the best discriminator of physical conditions (see Fig. 4).

The H $\alpha$ model has just been finished, and detailed comparisons to observations in specific binaries are beginning. We hope to identify excitation mechanisms responsible for such strong emission in relatively cool disks, to estimate disk masses, to verify the existence of non-Keplerian rotation, to estimate disk kinetic temperatures, and perhaps even to gauge the contribution of viscous dissipation to disk temperatures. An approximate Non-LTE treatment of the OI 7774 multiplet is planned for the model.

We gratefully acknowledge the director and staff of Mount Laguna Observatory for allocations of observing time and excellent observing support, respectively. M. Senay is acknowledged for observing assistance, and, along with J. Robertson (SDSU) and D. Bell (UI), for assistance with spectral data reduction. We also acknowledge the National Science Foundation for partial support (Etzel: AST-8822790; Olson: AST-8822351). PBE thanks the AAS for a supplemental travel grant to attend this IAU Symposium.

\section{References}

Budding, E. 1984, Bull. Inf. Centre des Donnees Stellaires (Strasbourg) 27, 91 Etzel, P.B. 1986, Ph.D. dissertation, UCLA

Etzel, P.B. 1988, AJ 95, 1204

Etzel, P.B. 1990, in Active Close Binaries, ed. by C. Ibanoglu, (Kluwer, Dordrecht), 203 Joy, A.H. 1942, PASP 54, 35 (reprinted 1988, PASP 100, 157)

Kaitchuck, R.H., Honeycutt, R.K., and Schlegel, E.M. 1985, PASP 97, 1178 
Olson, E.C. 1991, AJ 102, 1423

Peters, G.J. 1989, SSR 50, 9

Polidan, R.S., and Peters, G.J. 1976, in Be and Shell Stars, ed. by A. Slettebak (Reidel, Dordrecht), 59

Popper, D.M. 1989 ApJS 71, 595

Senay, M.C. 1991, M.S. thesis, SDSU

Slettebak, A. 1986, PASP 98, 867

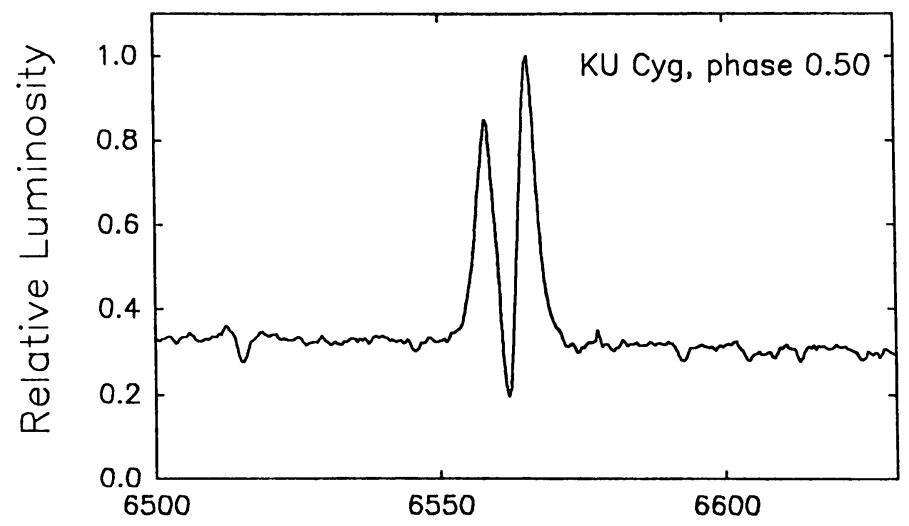

Fig. 1. - H $\alpha$ emission from KU Cyg. Double-peaked emission is visible throughout the entire orbital cycle.

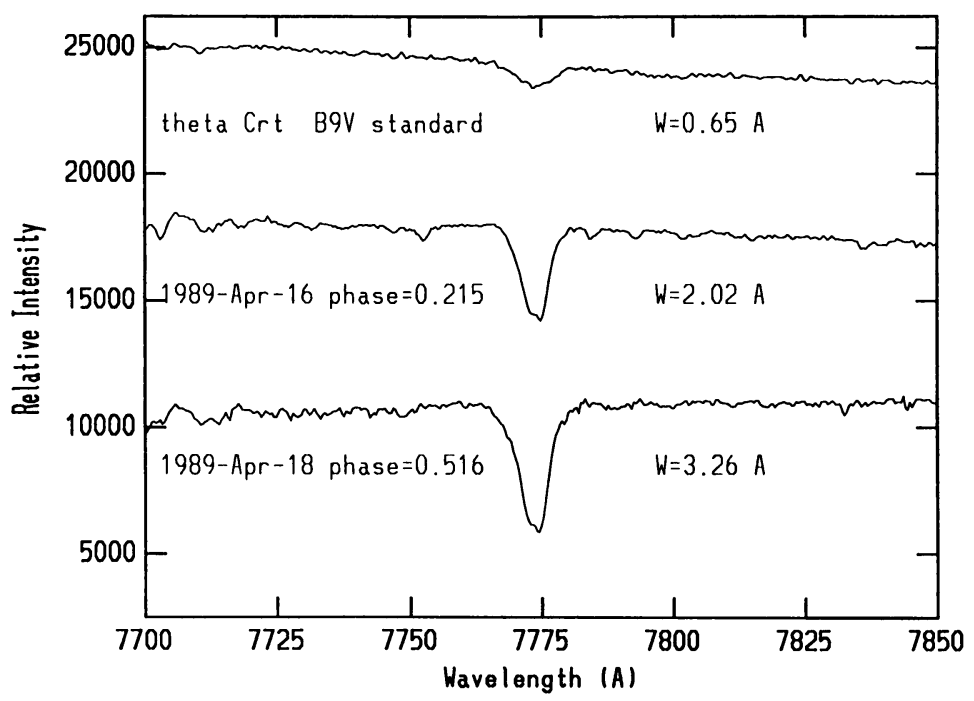

Fig. 2. - Demonstration of non-stellar nature of O I 7774 absorption in TT Hya, which contains a stable accretion disk. The hotter star in the system is similar to the spectral standard $\theta$ Crt. At quadrature phases, the disk partially occults the hotter component. At phases near secondary eclipse, the disk occults the cooler component to various degrees. 


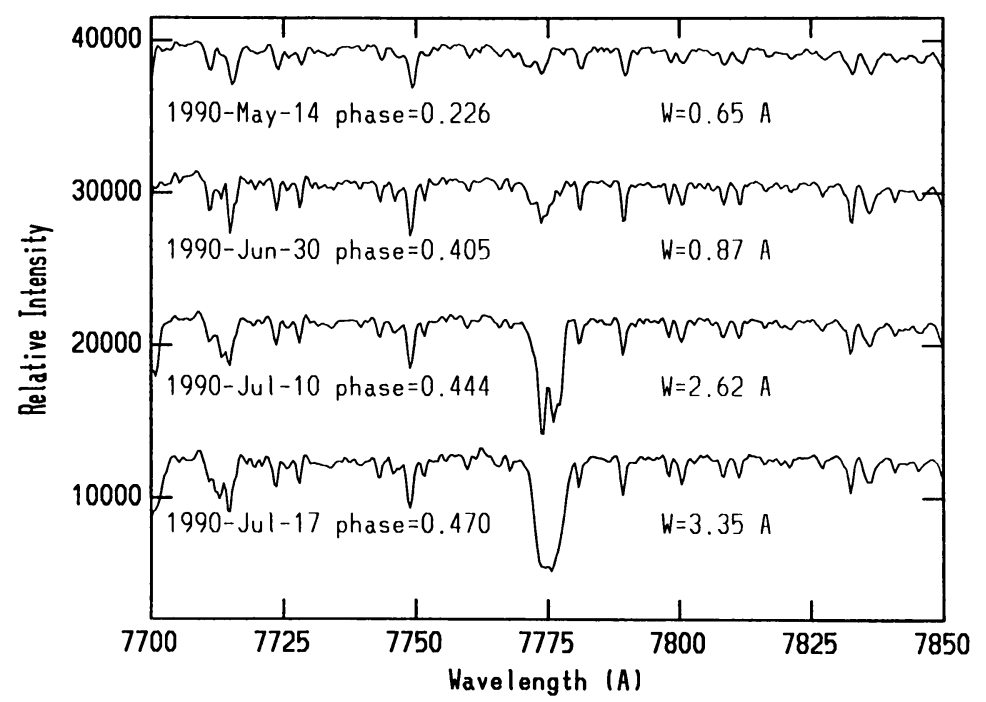

Fig. 3. - Variation of the O I 7774 absorption in RZ Oph. At this wavelength, the light of the two components is approximately equal. Absorption strength increases rapidly near secondary eclipse as the disk eclipses the much larger, mass-losing component.

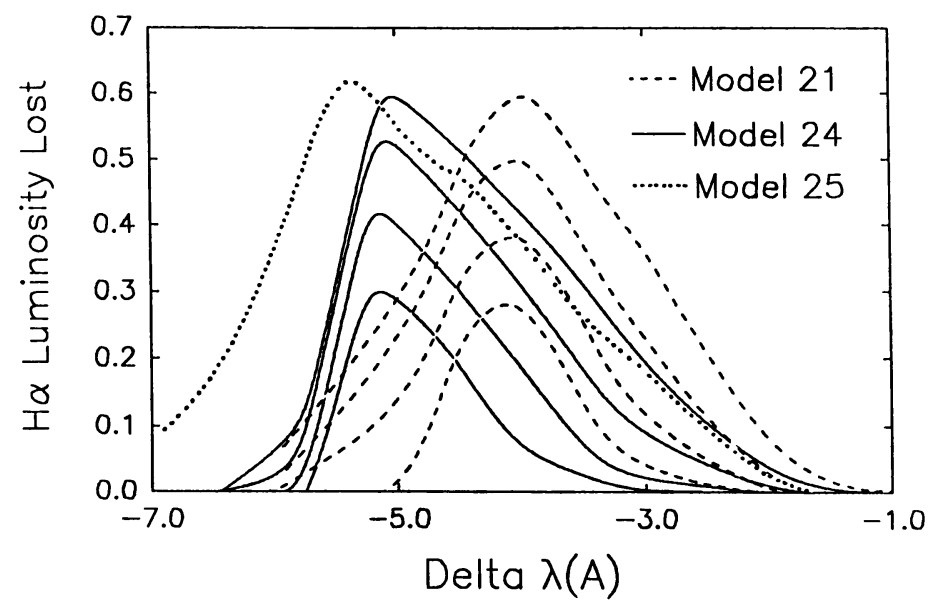

Fig. 4. - Theoretical non-LTE $H \alpha$ profiles of the light lost at various phases of the eclipse of the approaching lobe of the disk by the cool star. This profile set illustrates sensitivity to the disk rotation model. Model 21 is Keplerian; rotation in model 24 runs from $+30 \%$ to $-30 \%$ of Keplerian from the outer to the inner disk radius. Model 25 runs from $+40 \%$ of Keplerian to Keplerian. 